\title{
Centaurin- $\boldsymbol{\alpha}_{2}$ Interacts with $\boldsymbol{\beta}$-Tubulin and Stabilizes Microtubules
}

\author{
Paola Zuccotti ${ }^{19}$, Daniele Cartelli ${ }^{29}$, Michela Stroppi ${ }^{19}$, Vittorio Pandini ${ }^{2}$, Marco Venturin ${ }^{1}$, \\ Alessandro Aliverti ${ }^{2}$, Elena Battaglioli ${ }^{1}$, Graziella Cappelletti ${ }^{2 *}$, Paola Riva ${ }^{1 *}$
}

1 Dipartimento di Biotecnologie Mediche e Medicina Traslazionale, Università degli Studi di Milano, Milano, Italy, 2 Dipartimento di Bioscienze, Università degli Studi di Milano, Milano, Italy

\begin{abstract}
Centaurin- $\alpha_{2}$ is a GTPase-activating protein for ARF (ARFGAP) showing a diffuse cytoplasmic localization capable to translocate to membrane, where it binds phosphatidylinositols. Taking into account that Centaurin- $\alpha_{2}$ can localize in cytoplasm and that its cytoplasmatic function is not well defined, we searched for further interactors by yeast two-hybrid assay to investigate its biological function. We identified a further Centaurin- $\alpha_{2}$ interacting protein, $\beta$-Tubulin, by yeast twohybrid assay. The interaction, involving the C-terminal region of $\beta$-Tubulin, has been confirmed by coimmunoprecipitation experiments. After Centaurin- $\alpha_{2}$ overexpression in HeLa cells and extraction of soluble ( $\alpha \beta$ dimers) and insoluble (microtubules) fractions of Tubulin, we observed that Centaurin- $\alpha_{2}$ mainly interacts with the polymerized Tubulin fraction, besides colocalizing with microtubules (MTs) in cytoplasm accordingly. Even following the depolimerizing Tubulin treatments Centaurin- $\alpha_{2}$ remains mainly associated to nocodazole- and cold-resistant MTs. We found an increase of MT stability in transfected HeLa cells, evaluating as marker of stability the level of MT acetylation. In vitro assays using purified Centaurin- $\alpha_{2}$ and tubulin confirmed that Centaurin- $\alpha_{2}$ promotes tubulin assembly and increases microtubule stability. The biological effect of Centaurin- $\alpha_{2}$ overexpression, assessed through the detection of an increased number of mitotic HeLa cells with bipolar spindles and with the correct number of centrosomes in both dividing and not dividing cells, is consistent with the Centaurin- $\alpha_{2}$ role on MT stabilization. Centaurin- $\alpha_{2}$ interacts with $\beta$-Tubulin and it mainly associates to MTs, resistant to destabilizing agents, in vitro and in cell. We propose Centaurin- $\alpha_{2}$ as a new microtubule-associated protein (MAP) increasing MT stability.
\end{abstract}

Citation: Zuccotti P, Cartelli D, Stroppi M, Pandini V, Venturin M, et al. (2012) Centaurin- $\alpha_{2}$ Interacts with $\beta$-Tubulin and Stabilizes Microtubules. PLoS ONE 7(12): e52867. doi:10.1371/journal.pone.0052867

Editor: Yanmin Yang, Stanford University School of Medicine, United States of America

Received April 13, 2012; Accepted November 23, 2012; Published December 20, 2012

Copyright: (c) 2012 Zuccotti et al. This is an open-access article distributed under the terms of the Creative Commons Attribution License, which permits unrestricted use, distribution, and reproduction in any medium, provided the original author and source are credited.

Funding: This work was supported by an academic grant (PUR 2008) to PR and "Dote ricerca", FSE, Regione Lombardia to DC. The funders had no role in study design, data collection and analysis, decision to publish, or preparation of the manuscript.

Competing Interests: The authors have declared that no competing interests exist.

*E-mail: paola.riva@unimi.it (PR); graziella.cappelletti@unimi.it (GC)

9 These authors contributed equally to this work.

\section{Introduction}

Human Centaurin- $\alpha_{2}$, recently renamed "ARFGAP protein with dual $\mathrm{PH}$ (pleckstrin homology) domain-containing protein 2" $(A D A P 2)$ to stress the systematic relationships within the superfamily of ARFGAP proteins, is also characterized by a C4-type zinc finger and two $\mathrm{PH}$ domains. Centaurin- $\alpha_{2}$, together with $-\alpha_{1},-\beta,-\gamma$ and $-\delta$, constitutes the Centaurin family characterized by the presence of at least a $\mathrm{PH}$ domain, ankyrin repeats and a conserved $\mathrm{Cx}_{2} \mathrm{Cx}_{16-17} \mathrm{Cx}_{2} \mathrm{C}$ domain, which acts as a zinc-binding motif [1]. Centaurin- $\alpha_{2}$ has been shown to bind phosphatidylinositol-trisphosphate in vitro [2]. It has a diffuse cytoplasmic localization and has been found to translocate, after Epidermal growth factor (EGF) stimulation, from the cytoplasm to the plasma membrane, where it preferentially binds phosphatidylinositol-3,4-bisphosphate ( $\left.\mathrm{PIP}_{2}\right)$, through its $\mathrm{C}-\mathrm{PH}$ domain [3]. Centaurin- $\alpha_{2}$ displays a GTPase-activating protein activity on the ADP-ribosylation factor 6 (ARF6), a small GTPase involved in actin cytoskeleton remodelling. Plasma membrane association of Centaurin- $\alpha_{2}$ prevents ARF6 translocation to, and cortical actin formation at, the plasma membrane and thereby negatively regulates ARF6-mediated cytoskeleton actin reorganisation [3]. However the distribution of Centaurin- $\alpha_{2}$ in intact cells is predominantly cytosolic and, in this context, its biological function is not known.

Centaurin- $\alpha_{2}$ expression pattern reveals high expression levels in placenta, spleen, kidney, skeletal muscle and adrenal gland and weak signals in thyroid, liver, heart, lung, small intestine, peripheral blood leucocytes [2]. During human foetal development $A D A P 2$ was found to be expressed in skeletal muscle, liver and brain with a high expression in heart and aorta, and it has been detected in heart and brain during the first phases of mouse embryonic development [4]. It has been recently observed, by in situ hybridizations on mouse embryo, that Adap2 is expressed in early stages of heart development ( $9 \mathrm{dpc})$, during the formation of cardiac camera, septa and valves (M. Venturin, personal communication). Interestingly $A D A P 2$ gene was one of the genes found to be deleted in NF1 microdeletion patients showing a high incidence of cardiovascular malformations, most of which are valve or atrial/ventricular septa defects [5]. This evidence strongly suggests that $A D A P 2$ can be a candidate gene for these specific heart abnormalities. 
In an attempt to clarify the biological functions of this protein, we searched for cytosolic Centaurin- $\alpha_{2}$ interactors. Here we report on Centaurin- $\alpha_{2}-\beta$-Tubulin interaction, where Centaurin- $\alpha_{2}$ was found to be mainly associated to the $\beta$-Tubulin polymerized form increasing MT stability. Functional studies indicated that Centaurin- $\alpha_{2}$ stabilizes MTs with a role in the correct mitotic spindle formation. The obtained findings are strongly indicative that Centaurin- $\alpha_{2}$ is a new MAP.

\section{Results}

\section{Centaurin- $\alpha_{2}$ interacts with $\beta$-Tubulin}

With the aim of identifying novel proteins interacting with human Centaurin- $\alpha_{2}$, a yeast two-hybrid assay has been carried out using as bait a fusion protein between LexA DNA binding domain and the full length human Centaurin- $\alpha_{2}$. Following the exclusion of the auto-activation of $\beta$-galactosidase gene by the bait, avoiding false positives (see Materials and Methods), L40 yeast has been cotransformed with pSST91-Centaurin- $\alpha_{2}$ and a human foetal brain cDNA library. A screening of $1.2 \times 10^{6}$ cotransformants has led to the isolation of 36 positive clones growing on the selective medium -Leu - Trp - His positive at the $\beta$-galactosidase assay, in which two known interacting proteins CoRest-Kiaa0601 [6] have been used as positive control. The 36 positive clones have been shown to be unable to activate transcriptional machinery by means of $\beta$-galactosidase assay after extraction of the bait from transformed yeast. Following sequencing of each clone, six possible interactors have been identified. For all of them the interaction specificity for the Centaurin- $\alpha_{2}$-bait has been tested by using as baits unrelated control proteins, such as CoRest, Bars and Laminin. Only the construct encoding for Tubulin $\beta$ chain class I (NP_821133.1), showed a specific interaction with Centaurin- $\alpha_{2^{-}}$ bait (Figure $1 \mathrm{~A}$ ).

The Tubulin, $\beta$ cDNA has been found to encode for the last 116 aminoacids of the C-terminal protein region and to include part of the $3^{\prime}$ UTR. Tubulin $\beta$ chain class I isotype is ubiquitously expressed, sharing at least $95 \%$ of the aminoacid sequence with the other isotypes, with the exception of the class VI isotype sharing the $80 \%$ of the sequence. Tubulin $\beta$ chain, from now defined by the term $\beta$-Tubulin, is present in cells both in soluble dimeric form, associated with $\alpha$-Tubulin, and in polymerized form constituting MTs.

To confirm the interaction between Centaurin- $\alpha_{2}$ and Tubulin $\beta$, coimmunoprecipitation (CoIP) experiments have been carried out. HeLa cells, expressing low endogenous levels of Centaurin- $\alpha_{2}$, (Figure S1) were transfected with pCGN-Centaurin- $\alpha_{2}$, a vector encoding for HA (hemoagglutinin)-tagged Centaurin- $\boldsymbol{\alpha}_{2}$. Total cell lysates were immunoprecipitated with anti-HA antibody or a pre immune IgG serum (PI) and analyzed by western blotting. The anti-HA antibody identified a band with the apparent molecular weigth of $44 \mathrm{kDa}$ corresponding to the recombinant $\mathrm{HA}$ Centaurin- $\alpha_{2}$, both in the immunoprecipitation and in the input lanes (Figure $1 \mathrm{~B}$ ). Note that, due to cross-reaction of the HA antibody with the IgG, both in the IP and in the pre immune (PI) lane is present at about $50 \mathrm{kDa}$, a non specific band. After incubation with monoclonal anti- $\beta$-Tubulin antibody, a band of about $50 \mathrm{kDa}$ has been detected in IP lane as well as in the input lane (Figure $1 \mathrm{~B}$ ). The presence of a band corresponding to $\beta$ Tubulin in the HA-IP lane shows that Tubulin coimmunoprecipitates with Centaurin- $\alpha_{2}$, indicating that the interaction among Centaurin- $\alpha_{2}$ and $\beta$-Tubulin can occur in vivo in mammalian cells.

\section{Centaurin- $\alpha_{2}$ interacts with Tubulin preferentially associating with the polymerized Tubulin fraction in mammalian cells}

To establish if Centaurin- $\alpha_{2}$ interacts with Tubulin dimers or with MTs in cells, we analyzed cytoskeletal fractions obtained from pCGN-Centaurin- $\alpha_{2}$ transfected HeLa cells following Triton-X100 extraction. By western blotting and densitometric analysis, we evaluated the percentage of both Tubulin and Centaurin- $\alpha_{2}$ associated with Triton-soluble (S) and -insoluble (I) fraction. As shown in Figure $2 \mathrm{~A}$ and $\mathrm{B}$, Tubulin is similarly distributed between soluble (dimers) and insoluble (MTs) fractions as expected. Conversely, the greater part of Centaurin- $\alpha_{2}(80 \%)$ is found in the insoluble fraction, suggesting that Centaurin- $\alpha_{2}$ preferentially interacts with MTs, whereas the remaining $20 \%$ found in the soluble fraction could be associated with Tubulin dimers or could be free.

We therefore investigated if Centaurin- $\alpha_{2}$ binds to stable or labile MTs, resistant to destabilizing treatments or prone to depolymerization, respectively. To pursue this scope we challenged HeLa cells with well known MT-destabilizing treatments, such as nocodazole and cold. As expected, MTs significantly depolymerise following both treatments, and the vast majority of Tubulin is recovered in the dimeric fraction (Figure $2 \mathrm{~A}$ and $\mathrm{B}$ ). On the contrary, most of Centaurin- $\alpha_{2}$ remains associated with the Triton-insoluble fraction, highlighting that Centaurin- $\alpha_{2}$ binds to nocodazole- and cold-resistant MTs.

To confirm the Centaurin- $\alpha_{2}$ binding to Tubulin insoluble fraction, we transfected HeLa cells with pCGN-Centaurin- $\alpha_{2}$ or with the pCGN vector encoding only the HA-tag and removed the
A

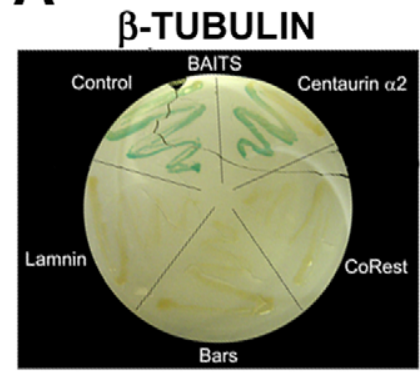

B

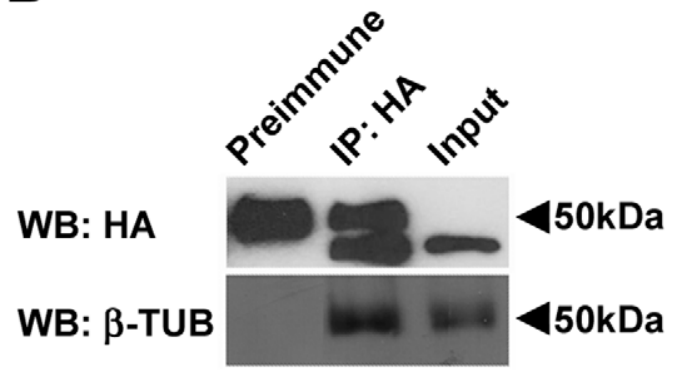

Figure 1. Centaurin- $\boldsymbol{\alpha}_{2}$ interacts with $\boldsymbol{\beta}$-Tubulin. A) Yeast two-hybrid assay on L40 yeast cotransformed with Tubulin $\beta$ chain and different baits (pSTT91-Centaurin- $\alpha_{2}$, pBTM116-CoRest, pBTM116-laminin or pBTM116-bars. B) Immunoprecipitation of $\beta$-Tubulin Centaurin- $\alpha_{2}$. Immunoblot of Centaurin- $\alpha_{2}$ (HA, upper panel) and of $\beta$-Tubulin ( $\beta$-Tub, lower panel) were performed on total extracts from HeLa cells transfected with pCGNCentaurin- $\alpha_{2}$ (Input), and on extracts immunoprecipitated with anti-HA antibody (IP:HA) and Preimmune serum. doi:10.1371/journal.pone.0052867.g001 
A

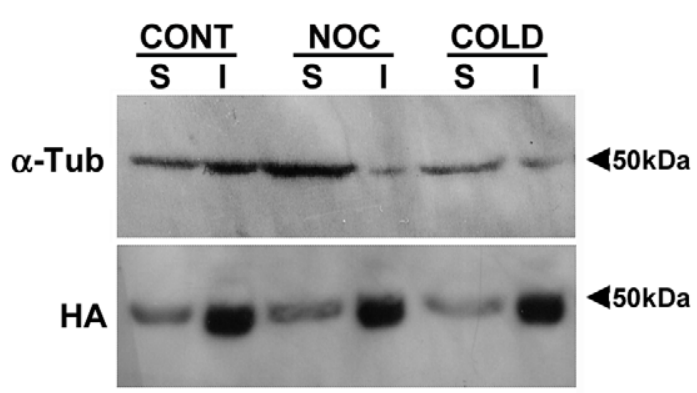

C
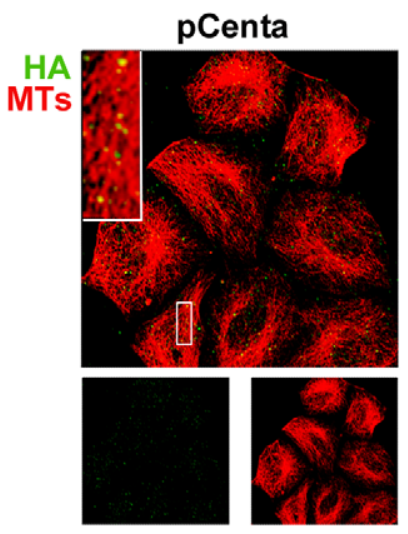

E

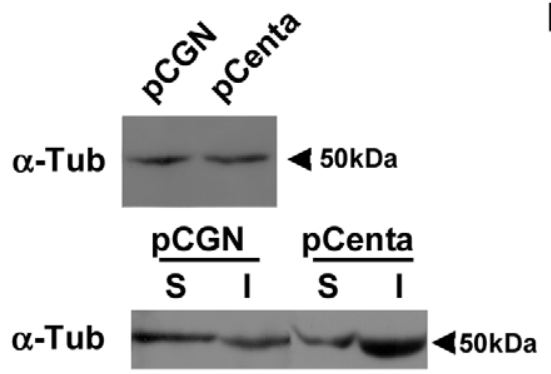

pCGN
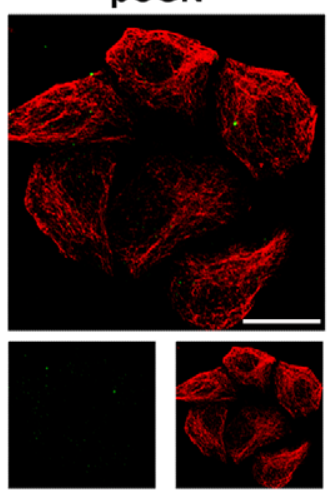

B

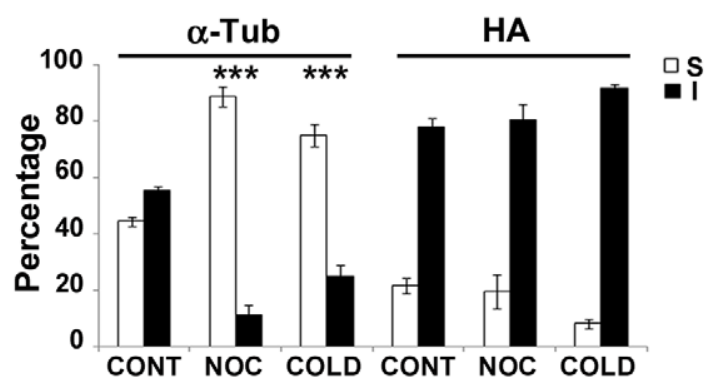

D
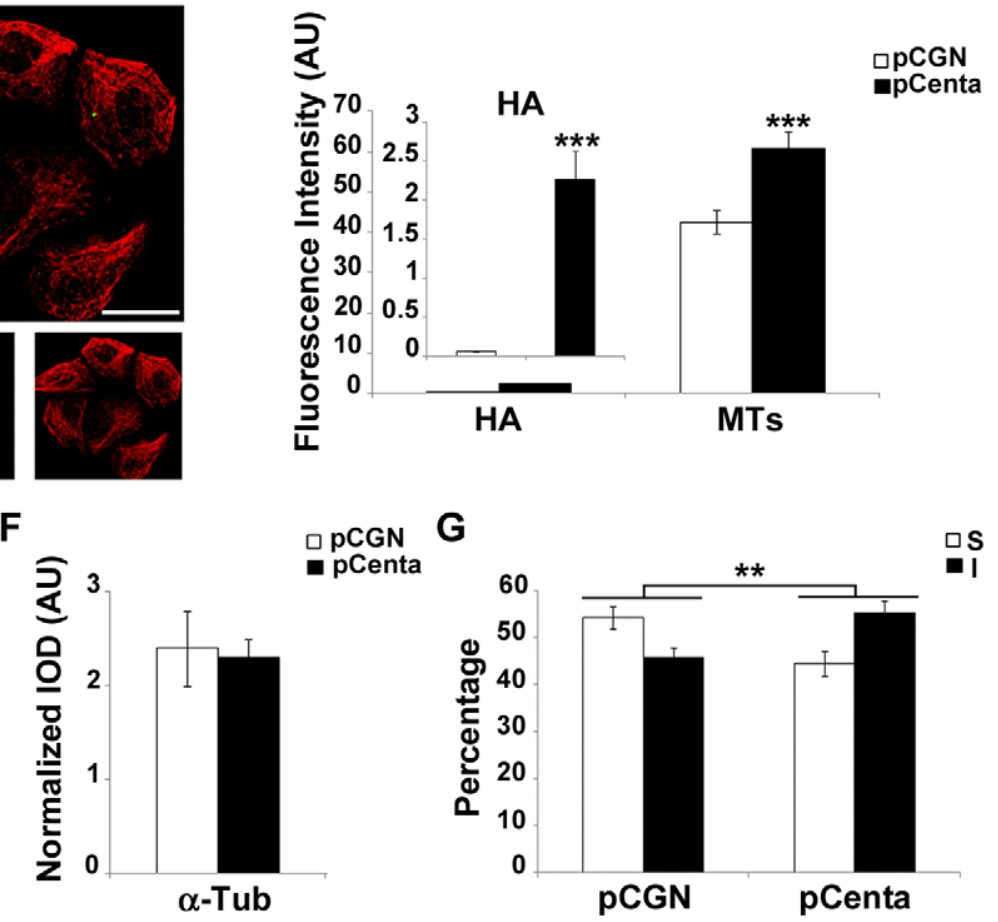

Figure 2. Centaurin- $\boldsymbol{\alpha}_{2}$ binds to stable MTs in HeLa cells. Immunoblots (A) and densitometric analyses (B) of Triton-soluble (S, white bars) and insoluble (l, black bars) fractions of pCGN-Centaurin- $\alpha_{2}$ transfected HeLa cells, in control conditions (CONT) or treated with nocodazole (NOC) and cold (COLD). ${ }^{* * *} p<0.005$ vs CONT. C) Cytoskeletal fractions of Hela cells transfected with pCGN-Centaurin- $\alpha_{2}$ or with pCGN vector were immonustained with anti-HA (green) and anti- $\alpha$-Tubulin (red) antibodies. For pCGN-Centaurin- $\alpha_{2}$ merged image, the inset (white rectangle) show the discrete distribution of HA spots along microtubules. Scale bar: $20 \mu \mathrm{m}$. D) Analyses of the intensity of fluorescence of green signal (HA) and red signal (MTs) in pCGN (white bars) or pCGN-Centaurin- $\alpha_{2}$ (black bars) expressing HeLa cells. ${ }^{* *} p<0.005$ vs pCGN. E) Immunoblots of the total and free (S) or polymerized (I) $\alpha$-Tubulin ( $\alpha$-Tub) and densitometric analyses of whole cell extracts (F) or Triton-soluble (S, white bars) and insoluble (I, black bars) fractions (G) of pCGN (pCGN) or pCGN-Centaurin- $\alpha_{2}$ (pCenta) transfected HeLa cells. ${ }^{* *} p<0.01$ vs pCGN. doi:10.1371/journal.pone.0052867.g002

Triton-soluble fraction. Then, we double stained the Tritoninsoluble fraction with anti- $\alpha$-Tubulin and anti-HA antibodies, and we analyzed the samples by confocal microscopy. As shown in Figure $2 \mathrm{C}$, there is no evidence of Centaurin- $\alpha_{2}$ aggregation; instead it appears as discrete punctae decorating MTs, meaning that Centaurin- $\alpha_{2}$ behaves like a MT interacting protein, remaining attached to MTs also after the removal of unassembled Tubulin. HeLa cells transfected with the control vector show very few HA spots, and the analyses of fluorescence intensity (Figure 2 D) report a significant lower HA fluorescence in pCGN transfected cells than in Centaurin- $\alpha_{2}$ expressing HeLa, allowing us to exclude a tag-mediated binding and supporting the in cell interaction between Centaurin- $\alpha_{2}$ and MTs. These results are reinforced by the analyses of colocalization parameters (reviewed in [7]).
The Pearson's coefficient (Table 1) together with the fluorograms (Figure S2) are rough estimations of the association between the fluorescence signals, and therefore they could show the association between the two proteins; indeed, our analyses reveal that the presence of Centaurin- $\alpha_{2}$ significantly increases the colocalization between $\mathrm{HA}$ and Tubulin signals reinforcing the idea of an interaction between the two proteins. Furthermore, the evaluation of Manders' coefficients (Table 1), representing the fraction of a signal superimposed to the other, highlights a significantly higher overlapping of both HA-Centa over Tubulin (M1) and Tubulin over HA-Centa (M2) compared to the empty vector (pCGN). Altogether, these analyses strengthen the idea of a direct binding of Centaurin- $\alpha_{2}$ to Tubulin in cell.

Interestingly, we observed that, Centaurin- $\alpha_{2}$ overexpressing cells show a significant increase in Tubulin fluorescence in respect 
Table 1. Analyses of colocalization between Centaurin- $\alpha_{2}$ and MTs.

\begin{tabular}{llll}
\hline & & & \\
\hline & pCGN & pCenta & $p$ Value \\
\hline Pearson & $0.017 \pm 0.003$ & $0.164 \pm 0.063$ & $<0.05$ \\
M1 (HA vs Tub) & $0.61 \pm 0.03$ & $0.71 \pm 0.02$ & $<0.03$ \\
M2 (Tub vs HA) & $0.01 \pm 0.003$ & $0.21 \pm 0.05$ & $<0.001$ \\
\hline $\begin{array}{l}\text { The } p \text { values are calculated according to the Student's t-test. } \\
\text { doi:10.1371/journal.pone.0052867.t001 }\end{array}$ &
\end{tabular}

to control cells. To verify if the higher Tubulin signal was due to an increased MT mass, we performed western blot analysis after extraction of total protein and of soluble/insoluble fractions in both pCGN-Centaurin- $\alpha_{2}$ and pCGN transfected HeLa cells. The obtained results indicate that Centaurin- $\alpha_{2}$ overexpression does not induce any increase of total Tubulin level (Figure $2 \mathrm{E}$ and F), while the distribution of Tubulin significantly shifts from the soluble to the insoluble pool in Centaurin- $\alpha_{2}$ transfected cells compared to the control (Figure $2 \mathrm{E}$ and $\mathrm{G}$ ). Taken together, these results suggest that Centaurin- $\alpha_{2}$ binding to MTs promotes the increase of the MT fraction and concomitant depletion of the free Tubulin pool, suggesting that Centaurin- $\alpha_{2}$ enhances MT stability.

\section{Pure Centaurin- $\alpha_{2}$ increases microtubule formation and stability in vitro}

To better characterize the interaction between Centaurin- $\alpha_{2}$ and MTs, we moved to experiments with pure proteins, performing polymerization of bovine brain tubulin in the presence of His-tagged Centaurin- $\alpha_{2}$ (Figure 3). At the end of the assembly, tubulin dimers $(\mathrm{S})$ and MTs $(\mathrm{P})$ were separated by centrifugation and resolved on SDS-PAGE and, as shown in Figure $3 \mathrm{~A}$, Centaurin- $\alpha_{2}$ alone is almost completely recovered in the supernatant whereas, in the presence of Tubulin, $80 \%$ of Centaurin- $\alpha_{2}$ is associated to MT fraction (Figure $3 \mathrm{E}$ ). Therefore, this approach allowed us to demonstrate that Centaurin- $\alpha_{2}$ mainly interacts with MTs. To verify whether true MTs are forming in the presence of Centaurin- $\alpha_{2}$, we used DIC microscopy. Figure 3 C (top) shows that Centaurin- $\alpha_{2}$ promotes the formation of MTs and, as revealed by anti-His immunofluorescence, it is associated to MTs. Following MT-destabilization by cold treatment (30 minutes of incubation on ice), we investigated if Centaurin- $\alpha_{2}$ binds to stable or labile MTs. This approach strongly suggests that Centaurin- $\alpha_{2}$ protects MTs from cold treatment and that it remains associated to cold-resistant MTs (Figure $3 \mathrm{C}$, bottom); indeed, after 30 minutes on ice, around $50 \%$ of Centaurin- $\alpha_{2}$ is still recovered in the MT fraction (Figure $3 \mathrm{C}$ and E). Finally, to verify that pure Centaurin- $\alpha_{2}$ is able to promote MT formation and stabilization we used two different approaches (Figure $3 \mathrm{C}$ and D). We performed MT assembly assay using an amount of tubulin around the critical concentration $(\mathrm{Cc})$, i.e. the minimal concentration allowing MT formation (17.85 $\mu \mathrm{M}$ for our tubulin batch). In control conditions a low level of tubulin was present in the polymerized fraction $(\mathrm{P})$, whereas Centaurin- $\alpha_{2}$ strongly increased MT assembly (Figure $3 \mathrm{C}$ and D). Moreover, using standard concentration of tubulin and allowing MT disassembly (30 minutes of incubation on ice at the end of polymerization), we recovered a significant higher amount of MTs in the presence of Centaurin- $\alpha_{2}$, meaning that Centaurin- $\alpha_{2}$ protects MTs against cold-induced depolimerization. Altogether, these data show that Centaurin- $\alpha_{2}$ directly interacts with MTs, promoting MT formation and stabilization, in vitro and in cell as well.

\section{Centaurin- $\alpha_{2}$ increases microtubule acetylation}

Nocodazole- and cold-resistant MTs are the most stable ones, and MT stability is regulated by Tubulin posttranslational modifications. A typical marker of dynamic MTs is the tyrosinated form of $\alpha$-Tubulin that represents also the newly synthesized Tubulin, whereas more stable MTs are typically associated with the acetylation of $\alpha$-Tubulin [8]. Interestingly, a protein binding nocodazole resistant $\mathrm{MTs}$, named Dual specificity protein phosphatase CDC14B, is reported to enhance MT stability through the increase of Tubulin acetylation [9]. Similarly we investigated whether Centaurin- $\alpha_{2}$ mediated changes in MT stability through modulation of Tubulin posttranslational modifications.

A western blotting and densitometric analysis on total extracts of HeLa cells transfected with either pCGN-Centaurin- $\alpha_{2}$ or pCGN, have been carried out by using antibodies specific for acetylated Tubulin, tyrosinated Tubulin and total $\alpha$-Tubulin. We found that both tyrosinated and acetylated Tubulin increases in Centaurin $\alpha_{2}$ overexpressing cells (Figure 4 A and B), even if only the Tubulin acetylation level is significantly higher, while total Tubulin level is the same in both Centaurin- $\alpha_{2}$ overexpressing and control cells. This finding together with the observation of a higher level of acetylated MT fraction in pCGN-Centaurin- $\alpha_{2}$ transfected cells compared to the control (Figure $4 \mathrm{C}$ and D), is consistent with the above reported role of Centaurin- $\alpha_{2}$ in stabilizing MTs.

\section{Centaurin- $\alpha_{2}$ promotes the proper assembly of mitotic apparatus}

The control of MT stabilization is crucial for the functionality of MT machinery and the correct expression of its biological functions. It is reported that more stable MTs, i.e. more acetylated MTs, confer resistance to breakdown of the mitotic apparatus [10]. Given that Centaurin- $\alpha_{2}$ overexpression induced changes in MT stability, we wondered whether Centaurin- $\alpha_{2}$ was implicated in mitotic apparatus assembly. pCGN-Centaurin- $\alpha_{2}$ and pCGN transfected HeLa cells have been serum starved for 24 hours and stimulated with EGF for 0, 1, 4, 16 minutes to induce proliferation. After decoration of MTs with anti- $\alpha$-Tubulin antibodies and chromosomes with DAPI, transfected and control cells show normal bipolar spindles but also aberrant forms, such as multipolar, characterized by plus than two centrosomes, and monopolar, with MTs radiating toward chromatin organized in a rosette pattern and a central polar area containing centrioles [11] (Figure $5 \mathrm{~A}$ ). Both in stimulated and non stimulated cells an increasing of bipolar spindles in Centaurin- $\alpha_{2}$ overexpressing cells compared to control was detected with a statistically significant difference after 4 and 16 minutes of EGF stimulation (Figure $5 \mathrm{~B}$ ). To verify whether the Centaurin- $\alpha_{2}$ downregulation lead to a further increase of abnormal mitotic spindle, HeLa cells were transfected with $A D A P 2$ specific-siRNA. The $A D A P 2$ mRNA level decreased up to $41 \%$ at 48 hours after cell transfection with $50 \mathrm{nM} A D A P 2$ siRNA. According to the treatments previously applied on Centaurin- $\alpha_{2}$ overexpressing cells, the number of abnormal spindle significantly increased after 16 minutes of EGF stimulation in respect to the control cells transfected with scrambled siRNA sequences (Figure $5 \mathrm{G}$ ). To elucidate the role of Centaurin- $\alpha_{2}$ in controlling the mitotic apparatus assembly we also evaluated the centrosome number in HeLa cells overexpressing Centaurin- $\alpha_{2}$ stimulated with EGF, both in interphase and mitosis. After a costaining with $\gamma$-Tubulin antibody and DAPI (Figure $6 \mathrm{~A}$ ) we found that the number of centrosomes significantly decreases in Centaurin- $\alpha_{2}$ overexpressing cells compared to the control, both in interphase and in metaphase after EGF stimuli (Figure 6 B). 
A

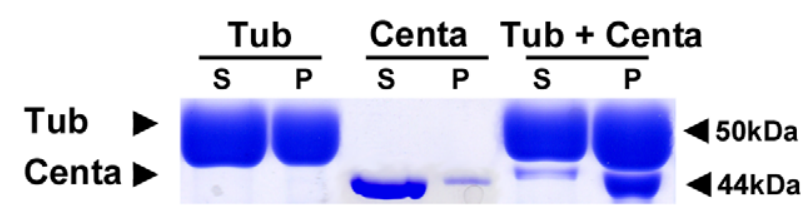

B

Tub

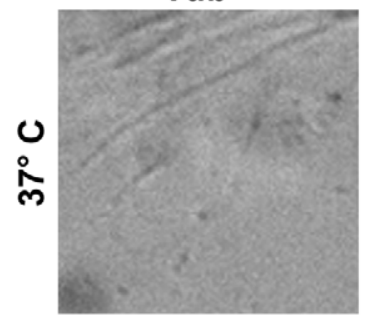

\begin{tabular}{l}
0 \\
\multirow{+}{*}{} \\
1 \\
0 \\
0 \\
0 \\
$m$
\end{tabular}

C

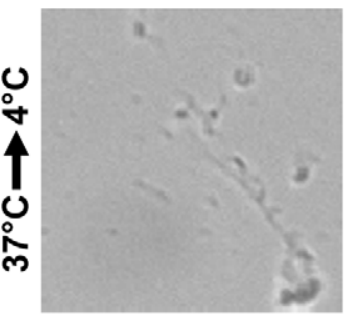

Tub + Centa
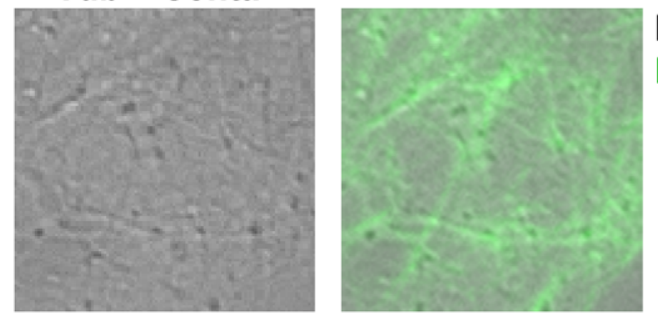

MTs

His
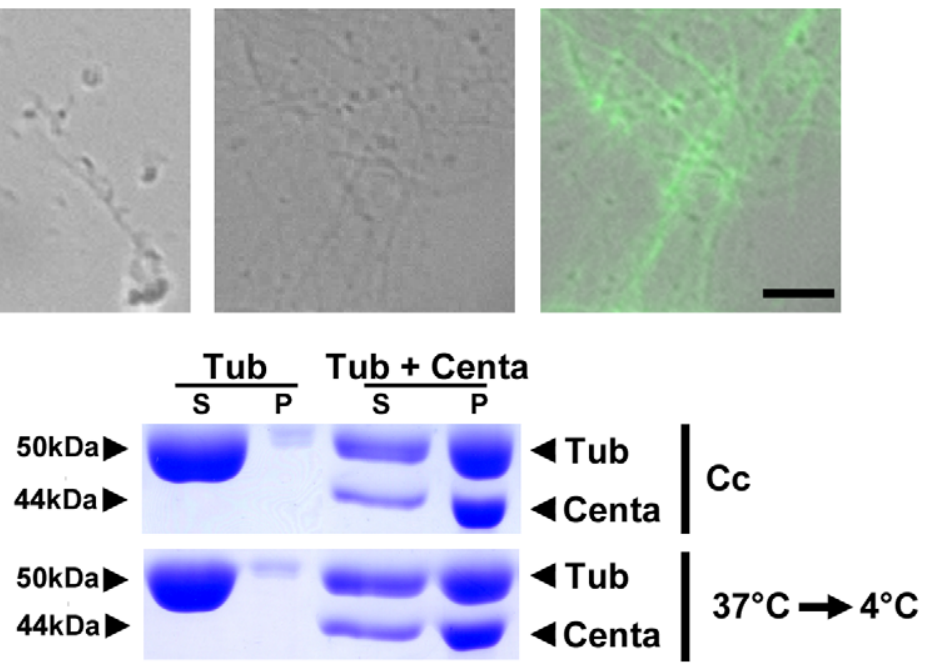

D

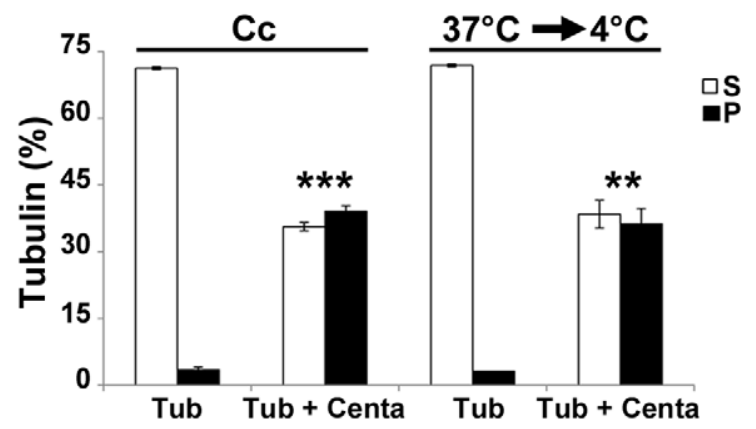

E

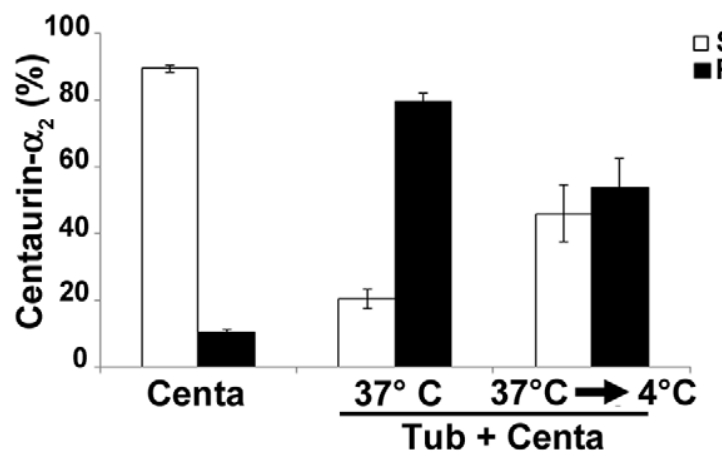


Figure 3. Centaurin- $\boldsymbol{\alpha}_{2}$ increases MT formation and stability. A) Comassie Blue stained SDS-PAGE gel of supernatant (S) and pellet (P) fractions of $40 \mu \mathrm{M}$ Tubulin (Tub), $5 \mu \mathrm{M}$ Centaurin- $\alpha_{2}$ (Centa) or $40 \mu \mathrm{M}$ Tubulin plus $5 \mu \mathrm{M}$ Centaurin- $\alpha_{2}$ (Tub+Centa) after 90 minutes of polymerization at $37^{\circ} \mathrm{C}$. B) Anti-His staining (Green) on in vitro MTs assembled in the absence (Tub) or in the presence of $5 \mu \mathrm{M}$ Centaurin- $\alpha_{2}$ (Tub $+\mathrm{Centa)}$, w/o ( $\left.37^{\circ} \mathrm{C}\right)$ or with $\left(37^{\circ} \mathrm{C} \rightarrow 4^{\circ} \mathrm{C}\right) 30$ minutes of incubation on ice. MTs were visualized by DIC microscopy. Scale bar: $2 \mu \mathrm{m}$. Comassie Blue stained SDS-PAGE gel (C) and densitometric analyses of tubulin content (D) of supernatant $(S$, white bars) and pellet ( $P$, black bars) fractions of MTs polymerized at $18 \mu \mathrm{M}$ tubulin (Cc) or at $40 \mu \mathrm{M}$ and then destabilized 30 minutes on ice $\left(37^{\circ} \mathrm{C} \rightarrow 4^{\circ} \mathrm{C}\right)$, in the absence (Tub) or in the presence of $5 \mu \mathrm{M}$ Centaurin- $\alpha_{2}(\mathrm{Tub}+\mathrm{Centa})$. ${ }^{* *} p<0.02,{ }^{* * *} p<0.005$ vs Tub. E) densitometric analyses of Centaurin- $\alpha_{2}$ associate to supernatant (S, white bars) and pellet (P, black bars) fractions of $5 \mu \mathrm{M}$ Centaurin- $\alpha_{2}$ (Centa) or $40 \mu \mathrm{M}$ Tubulin plus $5 \mu \mathrm{M}$ Centaurin- $\alpha_{2}$ (Tub+Centa) after 90 minutes of polymerization at $37^{\circ} \mathrm{C}\left(37^{\circ} \mathrm{C}\right)$ or after 30 minutes of incubation on ice $\left(37^{\circ} \mathrm{C} \rightarrow 4^{\circ} \mathrm{C}\right)$.

doi:10.1371/journal.pone.0052867.g003

Together these data show that Centaurin- $\alpha_{2}$ is able to regulate the correct assembly of mitotic apparatus, probably with a role in centrosomes duplication and modulation of MT stability.

\section{Discussion}

Centaurin- $\alpha_{2}$ is an ARFGAP protein showing a diffuse cytoplasmic localization capable to translocate to membrane, where it binds phosphatidylinositols, and displaying a GTPaseactivating protein activity towards ARF6 [3]. Taking into account that Centaurin- $\alpha_{2}$ can localize in cytoplasm and that its cytoplasmatic function is not well defined, we searched for further interactors by yeast two-hybrid assay to investigate its biological function. Starting from a human foetal brain cDNA library, we identified the interaction between Centaurin- $\alpha_{2}$ and $\beta$ Tubulin by yeast two hybrid assay and confirmed it by coimmunoprecipitation, in vitro assays using purified proteins and confocal microscopy in cells overexpressing Centaurin- $\alpha_{2}$. Furthermore, we showed that Centaurin- $\alpha_{2}$ is mostly associated to MTs, and maintained such an interaction also after depolymerizing treatments, i.e. cold treatment, suggesting a strong binding to MTs.

The Tubulin $\beta$ interactor identified in this study is a $\beta$-Tubulin isotype expressed ubiquitously and at high levels in cells. As the prey clone contains the C-terminal portion of Tubulin $\beta$ chain, corresponding to the hypervariable part of the protein characterizing specific isotypes $[12,13,14]$, we were able to identify the $\beta$ Tubulin isotype and in particular the region interacting with Centaurin- $\alpha_{2}$. The isolated prey clone encodes the last 116 aminoacids of the $\mathrm{C}$-terminal region, including two $\alpha$-helices, $\mathrm{H} 11$ and $\mathrm{H}_{12}$, that are known to constitute the external surface of MTs involved in the binding with motor proteins and MAPs (microtubule associated proteins) and seems to interact with the $\alpha$-Tubulin subunit $[13,14]$. Further possible Centaurin- $\alpha_{2}$ interactors have been reported: ARF6 [3], Elongation factor 1-gamma $[15,16]$, RanBPM (Ran binding protein in microtubule-organizing center) [17], Nucleolin [17]. All these proteins with the exception of the Elongation factor 1-gamma have been proposed as "candidate interactors" as they were demonstrated to bind Centaurin- $\alpha_{1}[16,17,18]$, showing a high sequence homology with Centaurin- $\alpha_{2}$ [19]. As far as the Elongation factor 1-gamma, it has been identified by an automated yeast two-hybrid assay, where the pool of preys did not include the Tubulin $\beta$ chain isotype. On the other hand, the experimental approach used in our study did not allow to fish the above described Centaurin- $\alpha_{2}$ interactors probably due to their enzymatic nature and their consequent transient binding to Centaurin- $\alpha_{2}$, as in the case of ARF6 and
A

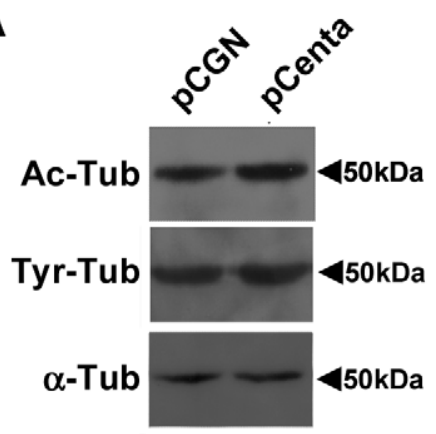

C

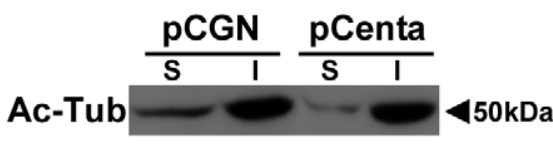

B
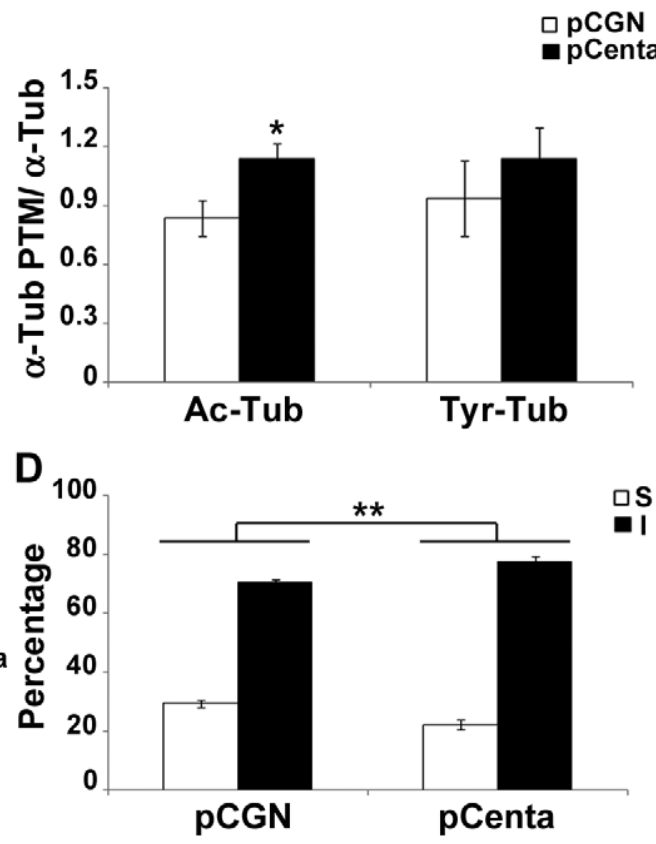

Figure 4. Centaurin- $\alpha_{2}$ increases MT acetylation. Immunoblot (A) and densitometric analyses (B) of acetylated- $\alpha$-Tubulin (Ac-Tub) and tyrosinated- $\alpha$-Tubulin (Tyr-Tub) in whole-cells extracts derived from HeLa cells transfected with pCGN (pCGN, white bars) or pCGN-Centaurin- $\alpha_{2}$ (pCenta, black bars). ${ }^{*} \mathrm{p}<0.05$ vs pCGN. Immunoblots (C) and densitometric analyses (D) of acetylated- $\alpha$-Tubulin (Ac-Tub) associated to Triton-soluble $\left(\mathrm{S}\right.$, white bars) and insoluble (l, black bars) of pCGN (pCGN) or pCGN-Centaurin- $\alpha_{2}$ (pCenta) expressing HeLa cells. ${ }^{* *} p<0.02$ vs pCGN. doi:10.1371/journal.pone.0052867.g004 
A BIPOLAR
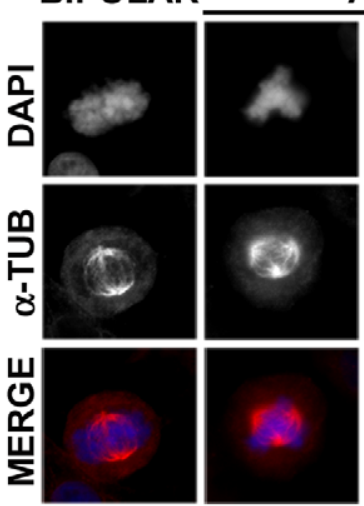

ABERRANT
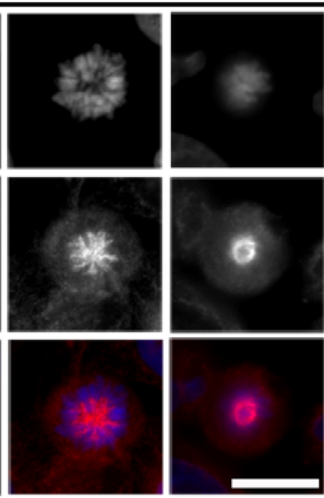

B

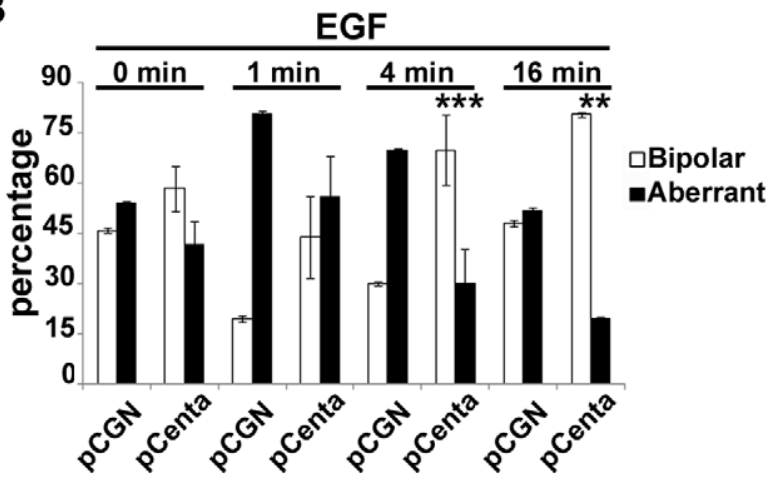

C

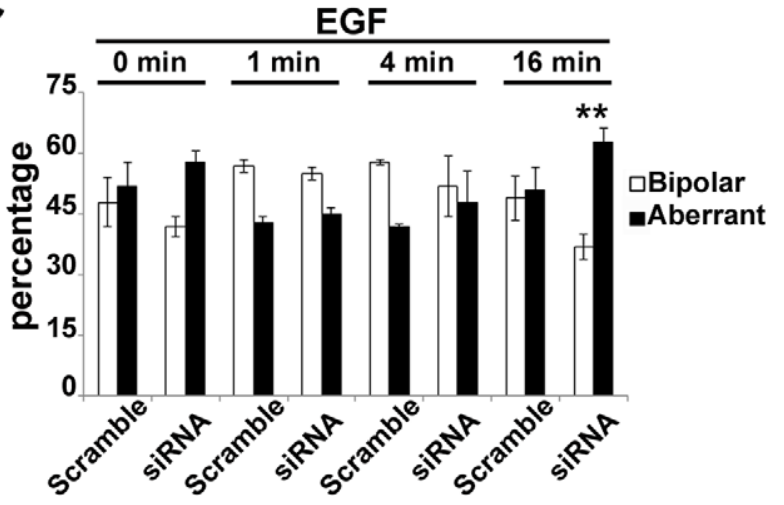

Figure 5. Centaurin- $\alpha_{2}$ promotes the correct assembly of mitotic apparatus. A) Representative microphotographs of the spindle morphologies observed in pCGN or pCGN-Centaurin- $\alpha_{2}$ expressing HeLa cells. B) Quantification of the percentage of cells displaying bipolar (white bars) or aberrant (black bars) spindles, after pCGN (pCGN) or pCGN-Centaurin- $\alpha_{2}$ (pCenta) transfection. C) Quantification of the percentage of cells displaying bipolar (white bars) or aberrant (black bars) spindles, after ON-TARGET plus SMART pool siRNA Centa2 or ON-TARGET plus Non-targeting Pool transfection, ${ }^{*} p<0.05$, ${ }^{* *} \mathrm{p}<0.02,{ }^{* * *} \mathrm{p}<0.005$ vs $\mathrm{pCGN}$. Scale bar $=20 \mu \mathrm{m}$. doi:10.1371/journal.pone.0052867.g005

Nardilysin $[3,16]$, or due to a restricted spatio-temporal binding, as in the case of RanBPM [17].

We found that Centaurin- $\alpha_{2}$ mostly binds to the polymerized Tubulin, resistant to destabilizing agents, indicating a strong association to stable MTs. This finding has been supported by data concerning the in vitro experiments and the co-localization of Centaurin- $\alpha_{2}$ and MTs after immunostaining and confocal microscopy analysis, indicating that it behaves like a MT
A

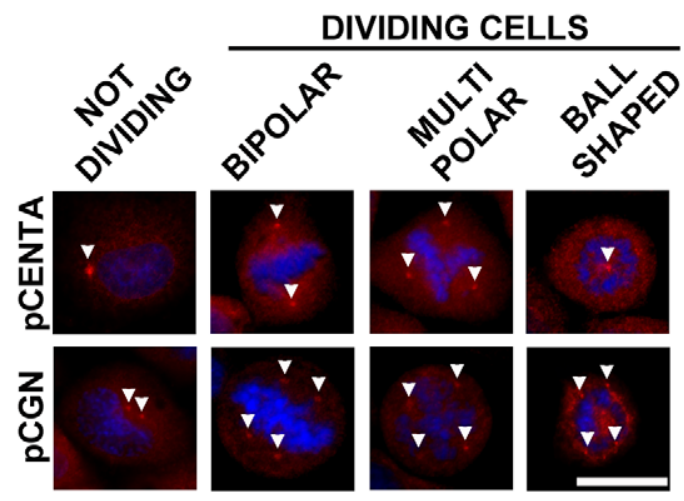

B

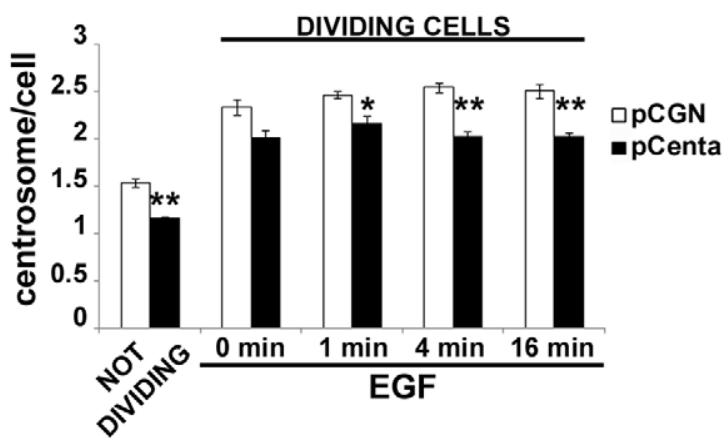

Figure 6. Centaurin- $\alpha_{2}$ decreases the HeLa cells with abnormal centrosome number. Representative microphotographs $(\mathbf{A})$ and quantification (B) of centrosomes numbers associate to the pCGN (pCGN, white bars) or pCGN-Centaurin- $\alpha_{2}$ (pCenta, black bars) expressing HeLa cells, in not dividing and dividing cells. ${ }^{*} p<0.05$, ${ }^{* *} \mathrm{p}<0.02$ vs $p C G N$. Scale bar $=20 \mu \mathrm{m}$. doi:10.1371/journal.pone.0052867.g006

interacting protein. Furthermore, the experiments with pure proteins revealed that Centaurin- $\alpha_{2}$ acts with a taxol-like mechanism, promoting MT assembly in constrained conditions, i.e. near tubulin critical concentrations, and protecting MTs from cold-induced destabilization. These data agree with the results we obtained in cell, indeed an increased amount of polymerized Tubulin has been detected both in vitro and at cellular level. Together, the interaction with the C-term portion of $\beta$-Tubulin, the binding to stable MTs and the ability in increasing MT assembly lead us to propose Centaurin- $\alpha_{2}$ as a new MAP. We speculated that the strong interaction between Centaurin- $\alpha_{2}$ and MTs might also be involved in the translocation of Centaurin- $\alpha_{2}$ from cytoplasm to plasma membrane by PIP $_{2}$ binding, after EGF stimulus [3]. Similarly phospholipase C- $\gamma_{1}\left(\mathrm{PL} \mathrm{C}-\gamma_{1}\right)$, which also contains a $\mathrm{PH}$ domain, was found to be constitutively associated with $\beta$-Tubulin and after EGF stimulation leads both proteins to move to membrane [20]. The results provided in both this study and in the previous work on PL C- $\gamma_{1}$, are consistent with the reported involvement of $\mathrm{PH}$ domains of signalling molecules in their targeted translocation to cell membrane [21].

Centaurin- $\alpha_{2}$ overexpression results in a higher amount of acetylated Tubulin that correlates with MT stability probably contributing to modulate intracellular trafficking. Indeed, MT acetylation speeds up synaptic vesicles transport and the inhibition of HDAC6, a MT deacetylases [22], leads to Kif5-cargo delocalization [23]. Furthermore, it is reported that Centaurin$\alpha_{1}$, that displays high homology with Centaurin- $\alpha_{2}$, interacts with $\mathrm{Kif13 \textrm {B }}$ and it is then transported to the leading edge of the cell. 
[24]. Taken together, these findings suggest a possible mechanistic explanation for the Centaurin- $\alpha_{2}$ translocation, namely the modulation of Tubulin/MT posttranslational modifications (PTMs). On the other hand, no MT PTM has been more frequently equated with MT stability than acetylation, especially the acetylation of lys 40 on $\alpha$-Tubulin, so much that the term "stable MTs" is often used almost synonymously with "Ac MTs" [8]. Nevertheless, Tubulin acetylation could be not the cause of MT stabilization [25] but it can occur on intrinsically more stable $\mathrm{MTS}_{\mathrm{s}}$, and both of them could be the cause or the consequence of intradimer conformational changes. Our results highlight that Centaurin- $\alpha_{2}$ interacts with cold- and nocodazole-resistant MTs and enhances acetylated-MTs in cell, but it directly stabilizes MTs in vitro. Therefore, we can propose that Centaurin- $\alpha_{2}$ directly contributes to the regulation of MT stability, particularly enhancing cold-adapted MT fraction, and to the exertion of the cell function that depends on acetylated Tubulin and stable MTs.

Although there is no a clear demonstration of the impact of acetylation of lys 40 of $\alpha$-Tubulin on microtubular and cellular functions, it is known that MT stability correlated to MT acetylation, confers resistance to breakdown of the mitotic apparatus [10]. Given that Centaurin- $\alpha_{2}$ overexpression has been demonstrated to induce changes in MT stability, an effect on mitotic apparatus assembly was expected. The obtained results support this hypothesis as we observed a decreasing number of Hela cells showing aberrant mitotic spindles, when Centaurin- $\alpha_{2}$ is overexpressed, while the cell number increases when $A D A P 2$ gene is downregulated, even if the moderate endogenous expression of Centaurin- $\alpha_{2}$ in HeLa cells probably does not allow to appreciate a high deviation of the number of normal/abnormal spindle in the siRNA treated in relation to control cells. The primary function of centrosomes is to nucleate and anchor MTs, meaning they play a key role in the establishment of the interphasic MT network and bipolar mitotic spindles. Looking at centrosomal proteins involved in controlling centrosome function, several MAPs have been implicated in MT dynamics regulation [26,27]. Among the others, Centrobindin is a centrosomal protein working as a MT stabilizing factor, able to promote MT formation, required for proper spindle formation during mitosis, and leading to defects in mitotic spindle when lacking [27]. Interestingly, our findings are consistent with such a peculiar role showing that Centaurin- $\alpha_{2}$ is a MAP, increases MT stability, and promotes the proper assembly of mitotic apparatus. Furthermore, the direct interaction of Centaurin- $\alpha_{2}$ with RanBPM [17], a known centrosomal protein [28], and the significant decreased number of centrosomes in Centaurin- $\alpha_{2}$ overexpressing cells, here reported, suggest its potential role in the control of centrosome formation. The integrity of centrosomes as well as their duplication are finely controlled by several factors including multiple kinases and oncogene activities [29,30,31]. In agreement with our results, a recent paper [32] suggests that modulation of MT stability is a regulatory element in the control of centrosome separation showing that the destabilization of interphase MTs speeds up this process. Thus, we propose that the Centaurin- $\alpha_{2}$-dependent stabilization of MTs contributes to the proper control of centrosome integrity and avoids centrosome amplification and the consequent formation of aberrant mitotic spindles. The presence of an aberrant number of centrosomes is reported in different tumor cells both in vitro and in vivo $[33,34]$ affecting mitotic spindle formation [29] and chromosome segregation for the presence of multinucleated cells [31] or a chromatin aggregation with one centrosome inside [11]. The occurrence of multipolar or the monopolar spindles as well as the aberrant number of centrosomes observed in HeLa and in other tumor cell lines, might be caused by a mutation and/or expression dysregulation of one or more genes encoding MAPs. The overexpression of Centaurin- $\alpha_{2}$ seems to show a protective effect on this aberrant condition by stabilizing MTs. Interestingly CENTA2 gene, alias ADAP2, was found to be expressed in neurofibromas and it has been candidate as a modifier gene contributing to a neurofibroma aberrant growth, both in number and in size, detected in NF1 microdeletion patients carrying a CENTA2 constitutional deletion [35]. Thus our results might address studies aimed at evaluating expression of Centaurin- $\alpha_{2}$ in tumors also suggesting to consider this protein as a new pharmacological molecule or target.

The expression of Centaurin- $\alpha_{2}$ during embryo development in central nervous system and in heart [4], might be related to the control of cytoskeleton stability during cell differentiation, shaping and migration. MT-based cytoskeleton is the primary spatial regulator of cell shape, and the coordinated action of Centaurin- $\alpha_{2}$ and its interactors might mediate changes in MT arrangement and MT-dependent function. Since RanBPM, which interacts with Centaurin $\alpha_{2}$ is also found to be associated with Integrin at the plasma membrane [36], the coordinate function of Centaurin- $\alpha_{2}$ and RanBPM could promote the nucleation and polymerization of MTs at centrosomes, and could increase the stiffness of molecular scaffold at the point of cellular adhesion, mediating changes in cell shape. MT- and actin-based cytoskeleton act in concert to orchestrate cell remodelling; moreover, one MT function is to shape Actin cytoskeleton at specific location, i.e. cell cortex, through the transport or activation/inactivation of Actin regulators [37]. In the light of these results, we hypothesize that Centaurin- $\alpha_{2}$ behaves as cytoskeleton cross-talker since it interacts with MTs stabilizing them, as we showed here, and regulates actin reorganization via ARF6 [3]. Consequently, Tubulin and Centaurin- $\alpha_{2}$ interaction not only increases MT stability but it can also serve to modulate Actin reorganization, regulating the global cellular morphology. Furthermore, fine regulation of cytoskeleton could be relevant from a pathogenic point of view. We thus speculate that haploinsufficiency or loss of function mutations of ADAP2 might affect MT stability and function of those cells, such as myocytes and neurons, in which the impairment of cytoskeleton organization, may lead to the onset of cardiovascular malformation and/or cognitive defects in NF1 microdeletion syndrome or in specific congenital diseases [5].

Evidence here provided on the binding between Centaurin- $\alpha_{2}$ and Tubulin- $\beta$ C-ter region, which has been demonstrated to interact with MAPs $[13,14]$, and the increased MT stability detected after Centaurin- $\alpha_{2}$ overexpression, mimicking a typical MAPs' function [38], strongly indicates that Centaurin- $\alpha_{2}$ may be a novel MAP. Further studies aimed at investigating its function in tumoral cells and its role during development, may elucidate its physiological role together with its implication in both tumorigenesis and in the onset of some congenital diseases.

\section{Materials and Methods}

\section{Yeast two-hybrid assay}

Yeast two-hybrid assay has been carried out on S. cerevisiae L40, grown in yeast peptone dextrose medium (20 g/l tryptone, $10 \mathrm{~g} / \mathrm{l}$ yeast extract) with dextrose (Clontech, Mountain View, CA) added with adenine hemisulfate $(100 \mathrm{mg} / \mathrm{l})$ (Sigma-Aldrich, St. Louis, MO). The bait, expressing Centaurin- $\alpha_{2}$, has been generated following PCR amplification of ADAP2 cDNA - Image 5214358 gene - service -, with primers designed to introduce proper restriction site for BamHI, and then subcloned in pSTT91 vector, in frame with DNA binding domain sequence. To verify the occurrence of bait auto-activation, that should be excluded, L40 
yeast has been co-transformed by lithium acetate in presence of polyethylene glycol solution $(50 \%$ polyethylene glycol 3350 (Sigma-Aldrich), 10X Tris-EDTA, 10X lithium acetate) with the bait construct pSTT91-Centaurin- $\alpha_{2}$ and the pACT2 empty vector. Colonies grown on selective medium -Leu - Trp have been tested for the $\beta$-galactosidase assay, as well as the positive control L40 yeast transformed with two interacting proteins CoRestKia0601 (Figure S3). L40 has been transformed using $240 \mu \mathrm{g}$ of pSST91-Centaurin- $\alpha_{2}$ ), $180 \mu \mathrm{g}$ of human fetal brain cDNA library (Matchmaker, Clontech) (made by $3.5 \times 10^{6}$ independent clones and constituted by cDNAs cloned in pACT2 vector using EcoRI and XhoI restriction enzimes, producing a fusion protein with regulatory protein GAL4), $600 \mu \mathrm{g}$ of salmon sperm. The transformation has been plated on -Leu - Trp - His plates (yeast nitrogen base (6.7 g/l) (Sigma-Aldrich), 50\% glucose, -Leu - Trp His $(0.62 \mathrm{~g} / \mathrm{l})$ (Clontech Laboratories Inc.) and agar $(15 \mathrm{~g} / \mathrm{l})$ for 2 3 days at $30^{\circ} \mathrm{C}$. Grown colonies have been screened for $\beta$ galactosidase activity using $67 \% \mathrm{X}-\mathrm{Gal}(20 \mathrm{mg} / \mathrm{ml}) / \mathrm{Z}$ buffer $\mathrm{pH} 7$ (16.1 g/l Na $2 \mathrm{HPO}_{4} 7 \mathrm{H}_{2} 0 ; 5.5 \mathrm{~g} / 1 \mathrm{NaH}_{2} \mathrm{PO}_{4} \mathrm{H}_{2} \mathrm{O} ; 0.75 \mathrm{~g} / \mathrm{l} \mathrm{KCl}$; $\left.0.246 \mathrm{~g} / \mathrm{l} \mathrm{MgSO}_{4} 7 \mathrm{H}_{2} \mathrm{O}\right) / 0.27 \% \beta$-mercaptoethanol solution and nitrocellulose filters PROTRAN (Schleicher\&Schuell BA 85) by incubation at $30^{\circ} \mathrm{C}$, in presence of a positive control pBTM116CoRest and pACT2-Kiaa0601. From colonies positive to $\beta$ galactosidase assay, prey plasmids have been extracted using lysis buffer (2\% Triton-X-100, 1\% SDS, $100 \mathrm{mM} \mathrm{NaCl,} 10 \mathrm{mM}$ Tris pH 7.5, 1 mM EDTA), phenol:chloroform:isoamilic 25:24:1 and acid washed glass beads (G-8772, 425-600 $\mu \mathrm{m}$, Sigma-Aldrich) and used for electroporation of E. coli Top 10. The plasmid, extracted using the Pure Yield Plasmid Miniprep System (Promega, WI), has been sequenced using the Big Dye ${ }^{\text {TM }}$ Terminator Cycle Sequence Ready Reaction Kit (Applied Biosystem, Carlsbad, CA), under the manufacturer's conditions, and resolved on a 3100 ABI Prism Genetic Analyzer (Applied Biosystem). The obtained cDNA, identified by http://blast.ncbi. nlm.nih.gov/Blast.cgi, has been digested with EcoRI (New England Biolabs, Ipswich, MA) and XhoI (New England Biolabs) restriction enzymes for identification of its length. Identified preys have been cotransformed in L40 yeast with different baits not interacting with them, pBTM116-CoRest, pBTM116-Laminin and pBTM116-Bars, in which every cDNA is cloned in frame with DNA binding domain, plated on -Leucine - Tryptophan plates $(0.64 \mathrm{~g} / \mathrm{l})$ (Clontech Laboratories Inc) and incubated for 2-3 days at $30^{\circ} \mathrm{C}$; the specificity of interaction with Centaurin- $\alpha_{2}$ has been observed after $\beta$-galactosidase assay.

\section{Coimmunoprecipitation assay}

HeLa cells have been transfected with pCGN-Centaurin- $\alpha_{2}$ vector. After 48 hours cells have been washed with $10 \mathrm{ml}$ of chilly $1 \mathrm{X}$ PBS in ice, scraped and centrifuged 5 minutes at $4^{\circ} \mathrm{C}$ at $180 \mathrm{~g}$; the pellet has been resuspended in 5 volumes of lysis buffer (50 mM Tris $\mathrm{pH} 8,150 \mathrm{mM} \mathrm{NaCl}, 0.5 \mathrm{mM}$ EDTA, $10 \mathrm{mM}$ imidazole $\mathrm{pH} \mathrm{7,0.5 \%}$ Triton-X-100, 10\% glycerol, $0.5 \mathrm{mM}$ dithiothreitol, threo-2,3-dihydroxy-1,4-dithiolbutane, $1 \mathrm{X}$ protein inhibitor cocktail (Sigma-Aldrich), 1X phenylmethanesulfonyl fluoride), rotated 30 minutes at $4^{\circ} \mathrm{C}$ and centrifuged at $14000 \mathrm{~g}$ for 30 minutes at $4^{\circ} \mathrm{C}$. The supernatant protein amount has been evaluated by Bradford Biorad protein assay (Biorad, Hercules, CA). The preparation of CoIP sample has been carried out as follow: $2.5 \mathrm{mg}$ of total protein extracts have been added to $1.25 \mu \mathrm{g}$ of HA-probe (Y-11) rabbit polyclonal IgG (Santa Cruz Biotechnology, Santa Cruz, CA) in a final volume of $300 \mu \mathrm{l}$ of lysis buffer. The preimmune sample was made combining $0.5 \mathrm{mg}$ of total protein extracts, $1.25 \mu \mathrm{g}$ of rabbit IgG (Santa Cruz Biotechnology) in a final volume of $300 \mu \mathrm{l}$ of lysis buffer.
Forty $\mu$ of slurry beads Protein $\mathrm{G}$ agarose (Invitrogen) in curran's blocking buffer buffer $(10 \mathrm{mM} \mathrm{MgCl}, 100 \mathrm{mM} \mathrm{KCl}$, $1 \mathrm{mM} \mathrm{CaCl}, 10 \mathrm{mM}$ imidazole $\mathrm{pH} \mathrm{7,5 \%} \mathrm{BSA,} \mathrm{0.3 \%} \mathrm{Tween-20,}$ $0.02 \% \mathrm{Na}$ azide) have been added to the samples rotated at $4{ }^{\circ} \mathrm{C}$ over night After a centrifugation of 2 minutes at $280 \mathrm{~g}$ at $4^{\circ} \mathrm{C}$ to pull down beads, the pellet has been washed with $1 \mathrm{ml}$ of wash buffer $(50 \mathrm{mM}$ Tris $\mathrm{pH} 8,150 \mathrm{mM} \mathrm{NaCl}, 10 \mathrm{mM}$ imidazole pH 7, 0,1\% Triton-X-100, 5\% glycerol, $0.5 \mathrm{mM}$ dithiothreitol, threo-2,3-dihydroxy-1,4-dithiolbutane, $1 \mathrm{X}$ protein inhibitor cocktail (Sigma-Aldrich), 1X phenylmethanesulfonyl fluoride) by rotating samples slowly for 4 minutes and following a centrifugation of 1 minutes at $280 \mathrm{~g}$. Samples have been analyzed by $8 \%$ SDS-PAGE.

\section{Western blotting}

The fractionation of cellular proteins into a soluble pool and a cytoskeleton associated pool was performed on HeLa cells, eventually transfected with pCGN-Centaurin- $\alpha_{2}$ or pCGN vectors, according to [39]. Briefly, cells were rinsed twice in $85 \mathrm{mM}$ Pipes (pH 6.94), $10 \mathrm{mM}$ EGTA, $1 \mathrm{mM} \mathrm{MgCl}_{2}, 2 \mathrm{M}$ glycerol, $1 \mathrm{mM}$ phenylmethanesulfonylfluoride, $0.1 \mathrm{mM}$ leupeptin, $1 \mu \mathrm{M}$ pepstatin, $2 \mu \mathrm{g} / \mathrm{ml}$ aprotinin buffer, extracted for $10 \mathrm{~min}$ at room temperature with the same buffer containing $0.1 \%$ Triton-X-100. The extraction of nocodazole-treated cells was performed in buffer supplemented with nocodazole at the same concentration as the treatment to prevent repolymerisation of MTs during extraction. Similarly, cold-treated cells were extracted with buffer maintained at $4{ }^{\circ} \mathrm{C}$. After extraction, the Triton-X-100-soluble fractions were diluted 3:1 with $4 \mathrm{X}$ SDS-PAGE sample buffer. The insoluble material remaining attached to the dish was scraped into SDSPAGE sample buffer containing protease inhibitors.

For preparation of whole-cell extracts, HeLa cells, eventually transfected with pCGN-Centaurin- $\alpha_{2}$ or pCGN vectors, have been washed twice with $1 \mathrm{X}$ PBS and scraped into SDS-PAGE sample buffer containing protease inhibitors. The protein concentration has been determined by bicinchoninic acid reagent assay (Micro BCA, Pierce) and compared to a bovine serum albumin standard curve, to separate an equal amount of each sample.

Protein samples were separated by 7.5\% SDS-PAGE, Western blotted onto nitrocellulose membrane (Whatman protran BA 85, $0.45 \mu \mathrm{m}$ ) (for coimmunoprecipitation experiments) or onto polyvinylidene fluoride membranes (ImmobilonTM-P, Millipore). Nitrocellulose membranes have been blocked with 5\% BSA/1\% 100X Na Azide in Tris-buffered saline for 30 minutes at room temperature; polyvinylidene fluoride membranes blocking has been performed with $0.1 \mathrm{M}$ Tris- $\mathrm{HCl} / 0.9 \% \mathrm{NaCl} / 5 \% \mathrm{BSA} /$ $0.05 \%$ Tween-20 for 150 minutes at room temperature. The membranes have been probed over night at $4{ }^{\circ} \mathrm{C}$ with the following antibodies (Ab): diluted 1:200 HA-probe (Y-11) rabbit polyclonal IgG (Santa Cruz Biotechnology) or diluted 1:5000 monoclonal anti- $\beta$-Tubulin mouse IgG1 (clone TUB 2.1, Sigma-Aldrich) in Tris-buffered saline/5\% BSA/0.1\% Tween-20; diluted 1:1000 $\alpha$ Tubulin mouse IgG (clone B-5-1-2, Sigma-Aldrich), diluted 1:1000 Ac-Tubulin mouse IgG (clone 6-11B-1, Sigma-Aldrich) or diluted 1:1000 Tyr-Tubulin mouse IgG (clone TUB-1A2, Sigma-Aldrich) in $1 \mathrm{X}$ Tris-buffered saline $/ 1 \%$ BSA $/ 0.05 \%$ Tween- 20 . The incubation with secondary antibodies for $1 \mathrm{~h}$ at room temperature have been performed using the following antibodies: diluted 1:2000 ECL-rabbit IgG, HRP (horseradish peroxidise)-linked whole Ab from donkey (Amersham, Piscataway, NJ) or 1:1000 or goat anti-mouse IgG-HRP (Santa Cruz Biotechnology) in Trisbuffered saline $/ 5 \%$ BSA $/ 0.1 \%$ Tween-20 for $1 \mathrm{~h}$ at room temperature; diluted 1:40000 HRP goat anti-rabbit IgG (Pierce, Rockford, IL) or diluted 1:20000 HRP goat anti-mouse IgG 
(Sigma-Aldrich) in 1X Tris-buffered saline/1\% BSA/0.05\% Tween-20. Chemiluminescent signals have been detected using kit Supersignal West Pico Chemiluminescent Substract (Pierce), as instructed by the supplier. Protein quantification has been performed by scanning immunoblots with the JX-330 color image scanner (Sharp Electronics Europe) and analyzed by ImageJ software (National Institute of Health).

\section{Immunofluorescence and colocalization analyses}

After $48 \mathrm{~h}$ HeLa cells, transfected with pCGN-Centaurin- $\alpha_{2}$ or pCGN vectors, were fixed with $4 \%$ paraformaldehyde (SigmaAldrich) for 10 minutes at room temperature and permeabilized for $4 \mathrm{~min}$ with $0.1 \%$ Triton-X-100/1X PBS. Some slides were extracted before the fixation to remove unassembled Tubulin. Briefly, cells were washed twice with PEM (Pipes $80 \mathrm{mM}$, EGTA $5 \mathrm{mM}, \mathrm{MgCl}_{2} 1 \mathrm{mM}, \mathrm{pH} \mathrm{6,8}$, with protease inhibitors) and extracted 2 minutes with PEM buffer with Triton X-100 0,5\%, $\mathrm{NaCl}$ 0,2 M protease inhibitors and $10 \mu \mathrm{M}$ Paclitaxel (SigmaAldrich), and then fixed with methanol at $-20^{\circ} \mathrm{C}$. The samples have been treated with $5 \%$ BSA for 15 minutes at room temperature and incubated with diluted 1:200 HA-probe (Y-11) rabbit polyclonal IgG (Santa Cruz Biotechnology), with diluted 1:500 $\alpha$-Tubulin mouse IgG (clone B-5-1-2, Sigma-Aldrich) or with diluted 1:1000 $\gamma$-Tubulin mouse IgG (clone GTU-88, Sigma Aldrich) in Tris-buffered saline $/ 1 \% \mathrm{BSA}$ for $1 \mathrm{~h}$ at $37^{\circ} \mathrm{C}$. After washing twice with $1 \mathrm{X}$ PBS, samples have been incubated with diluted 1:1000 Alexa Fluor ${ }^{\text {TM }} 488$ goat anti-rabbit (Invitrogen) and diluted 1:1000 Alexa Fluor ${ }^{\mathrm{TM}} 594$ goat anti-mouse (Invitrogen) in Tris-buffered saline $/ 1 \%$ BSA for 45 minutes at $37^{\circ} \mathrm{C}$. Concurrent nuclear staining was made with 4',6-Diamidino-2phenylindole dihydrochloride (Sigma-Aldrich). The coverslips were mounted in Mowiol $^{\circledR}$ (Calbiochem)- DABCO (SigmaAldrich) and examined with an Axiovert $200 \mathrm{M}$ microscope (Carl Zeiss, Oberkochen, Germany) or with a confocal laser scan microscope imaging system (TCS SP2 AOBS, Leica Microsystems) equipped with an Ar-Ar/Kr488 nm, $561 \mathrm{~nm}$ and $405 \mathrm{~nm}$ diode lasers. Photomultiplier gain for each channel was adjusted to minimize background noise and saturated pixels and, once defined for control conditions, parameters were kept constant for all acquisitions; complete ' $z$ ' series optical sections were collected and projected onto a single plane using Leica TCS software. The analyses of colocalization parameters, reviewed in Bolte and Cordeliers (2006), have been made on the single-plane the raw images using the JACoP plug in for the Image J software.

Two different kinds of analyses were carried out: estimation of Pearson's coefficient, that represents the rough association between the two signals, and hence between two proteins, being the simpler way of measuring the dependence of pixels in dualchannel images, but particularly subjected to noise effect; evaluation of Manders' coefficients, that are good indicators of the relative distribution of the signals/proteins, representing the proportion of a signal (i.e. green) coincident with the other signal (i.e. red) over its total intensity, which may be applied even if the intensities in the two signals are really different.

\section{Recombinant centaurin- $\alpha 2$ production}

In order to generate a plasmidic construct for the synthesis of human centaurin- $\alpha 2$ in Escherichia coli, the insert of the pCGNbased construct was amplified by PCR using the two primers $5^{\prime}$ GGTGGTCATATGGGCGATCGCG-3' and 5'GGTGGT GGATCCCTTCTTCCAACAAGGAG-3', which in-

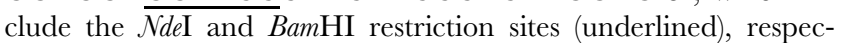
tively. After the restriction enzyme digestion, the resulting fragment was inserted between the same sites of the pET28b vector (Novagen), yielding the expression plasmid pET-Cent $\alpha 2$, whose insert region was fully sequenced to exclude the presence of PCR artifacts. For recombinant centaurin- $\alpha 2$ production, BL21(DE3) E. coli cells (Novagen) were cotransformed with pLacIRARE2 (Novagen) and pET-Cent $\alpha 2$, grown in liquid terrific broth supplemented with chloramphenicol and kanamycin, and induced overnight at $15^{\circ} \mathrm{C}$ with $0.1 \mathrm{mM}$ isopropyl- $\beta$-D-thiogalactopyranoside. The target protein, which carries an N-terminal His-tag extension, was isolated from the soluble fraction of the crude bacterial lysate by immobilized metal ion affinity chromatography on a His Trap HP $5 \mathrm{ml}$ prepacked column (GE Helthcare), according to the manufacturer directions. After concentration by ultrafiltration and desalting by gel filtration, the protein, showing higher than $95 \%$ purity by SDS-PAGE, was subdivided in aliquots and stored at $-70^{\circ} \mathrm{C}$ until needed.

\section{Tubulin purification and assembly assay}

Tubulin was purified from bovine brain purchased from a local slaughterhouse, conserved before use in ice-cold PIPES buffer (1 M K-PIPES, pH 6.9, $2 \mathrm{mM}$ EGTA, and $1 \mathrm{mM} \mathrm{MgCl}_{2}$ ) and used as soon as possible. Pure tubulin was obtained by two cycles of polymerisation-depolymerisation in a high-molarity buffer [40], and protein concentration was determined by the MicroBCA assay kit (Pierce). To remove the cold-stable fraction, just before the use, tubulin was centrifuged at $100000 \mathrm{~g}$ (Beckmann, rotor TLA 100.3) for 30 minutes at $4^{\circ} \mathrm{C}$. Assembly buffer $(80 \mathrm{mM} \mathrm{K}$ PIPES pH 6.9, $2 \mathrm{mM}$ EGTA, $1 \mathrm{mM} \mathrm{MgCl}_{2}$, and $10 \%$ glycerol) contained tubulin at two different concentrations, $40 \mu \mathrm{M}$ (standard conditions) or $18 \mu \mathrm{M}$ (around the critical concentration) and $5 \mu \mathrm{M}$ Centaurin- $\alpha_{2}$, and the polymerization run for 90 minutes at $37^{\circ} \mathrm{C}$. As control, tubulin was incubated with the same buffer containing Centaurin- $\alpha_{2}$. In some cases, after the assembly samples were kept on ice for 30 minutes, to allow microtubule depolymerisation. To separate microtubules (pellet) and tubulin dimers (supernatant), samples were centrifuged at $16500 \mathrm{~g}$ for 30 minutes at room temperature; equal amount of each fraction was resolved on 10\% SDS-PAGE gel and stained with Comassie Blue. Alternatively, at the end of the assembly, microtubules were fixed with $0.5 \%$ glutaraldehyde in BRB 80 and put onto poli-Llysine coated slides. Subsequently, the presence of His-tagged Centaurin- $\alpha_{2}$ was detected by using an anti-His primary antibody (IgG mouse, clone 4A12E4, Invitrogen) and Alexa Fluor ${ }^{\mathrm{TM}} 488$ goat anti-mouse (Invitrogen). The coverslips were mounted in Mowiol $^{\circledR}$ (Calbiochem, San Diego, CA)-DABCO (Sigma-Aldrich) and image acquisition was performed using a Zeiss Axiovert 200 equipped with differential interference contrast (DIC) optics and fluorescent filter units, a $63 \times$ oil objective, and a digital image recording system (Axiocam HRM Rev. 2 camera driven by Axiovision software rel. 4.4, Zeiss).

\section{Mammalian cell cultures and transfection}

HeLa cells have been cultured in Dulbecco's modified Eagle's medium with $10 \%$ fetal bovine serum, $100 \mathrm{U} / \mathrm{ml}$ penicillinstreptomycin and $0.01 \mathrm{mM}$ L-glutamine (EuroClone, Pero $\mathrm{MI}$ ). Cultures have been maintained at $37^{\circ} \mathrm{C}$ in a $5 \% \mathrm{CO}_{2}$ incubator. Cells have been transfected at $60-70 \%$ confluence and cultured at $37^{\circ} \mathrm{C}$ in a humidified atmosphere with $5 \% \mathrm{CO}_{2}$. Transfection has been performed with LIPOFECTAMINE 2000 (Invitrogen) according to the manufacturer's instructions using pCGN vector or pCGN-Centaurin- $\alpha_{2}$ (generated from PCR amplification of ADAP2 cDNA - Image 5214358 gene - service - with primers designed to introduce proper restriction site for BamHI and then by subcloning them in pCGN vector, in frame with HA sequence). 
In experiments using nocodazole $(3 \mu \mathrm{g} / \mathrm{ml})$ (Sigma-Aldrich) to depolymerize MTs, the drug was diluted into the culture medium and incubated for $3 \mathrm{~h}$ at $37^{\circ} \mathrm{C}$ prior to cellular fractionation for biochemical analyses. Cold treatments were performed at $4^{\circ} \mathrm{C}$ by incubating cultures in a refrigerator for $3 \mathrm{~h}$ prior to cellular fractionation for biochemical analyses.

\section{$A D A P 2$ silencing}

ADAP2 gene silencing has been carried out using $50 \mathrm{nM}$ ONTARGET plus SMART pool siRNA Centa2 (J-020444-09: gaguuaaaucugugcgacu; J-020444-10: aaguaugagagacgggaau; J020444-11: cuucaagcaccacgcagaa; J-020444-12: gauaugaagccuacgaaga) and ON-TARGET plus Non-targeting Pool (100 nM) (Thermo SCIENTIFIC, CO, USA) with LIPOFECTAMINE 2000 (Invitrogen, Carlsbad, CA), as described [41]. The total RNAs from both treated and non-treated cells were isolated at 24, 48 and 72 hours after the transfection using TRIzol reagent (Invitrogen), according to the manufacturer's instructions. Purity of RNAs (A260/A280 value of 1.8-2.1) and concentration were measured using Nanodrop spectrophotometer. To eliminate DNA contamination, total RNAs were treated with DNase I (Invitrogen). cDNA was synthesized from total RNA $(1 \mu \mathrm{g})$ in $20 \mu \mathrm{l}$ reactions, using iScript cDNA Synthesis Kit from BioRad (Hercules, CA, USA).

To estimate the mRNA amount a three-step real-time PCR analysis was performed, by using Gotaq qPCR sybergreen (Promega, WI), Centa 2 and HPRTI housekeeping primer forward and reverse (Centa2 fw acaccaggaacctgtttgtgt; Centa2 rev: gagggcattgaaccagtcc; and HPRTI fw: tgacactggcaaaacaatgca, HPRTI rev: ggtccttttcaccagcaagct).

\section{Statistical analysis}

The statistical significance of treatment has been assessed by Student's t-Test for parametric variables and by $\chi^{2}$ test for nonparametric ones, and analyses were performed using STATISTICA (StatSoft Inc., Tulsa, OK). All experiments have been repeated at least three times and data have been expressed as means \pm S.E.M (Standard Error of the Mean).

\section{References}

1. Cukierman E, Huber I, Rotman M, Cassel D (1995) The ARF1 GTPaseactivating protein: zinc finger motif and Golgi complex localization. Science 270: 1999-2002.

2. Hanck T, Stricker R, Sedehizade F, Reiser G (2004) Identification of gene structure and subcellular localization of human centaurin alpha 2 , and p42IP4, a family of two highly homologous, Ins 1,3,4,5-P4-/PtdIns 3,4,5-P3-binding, adapter proteins. J Neurochem 88: 326-336.

3. Venkateswarlu K, Brandom KG, Yun H (2007) PI-3-kinase-dependent membrane recruitment of centaurin-alpha2 is essential for its effect on ARF6mediated actin cytoskeleton reorganisation. J Cell Sci 120: 792-801.

4. Venturin M, Bentivegna A, Moroni R. Larizza L, Riva P (2005) Evidence by expression analysis of candidate genes for congenital heart defects in the NF1 microdeletion interval. Ann Hum Genet 69: 508-516.

5. Venturin M, Guarnieri P, Natacci F, Stabile M, Tenconi R, et al. (2004) Mental retardation and cardiovascular malformations in NF1 microdeleted patients point to candidate genes in 17q11.2. J Med Genet 41: 35-41.

6. Yang M, Gocke CB, Luo X, Borek D, Tomchick DR, et al. (2006) Structural basis for CoREST-dependent demethylation of nucleosomes by the human LSD1 histone demethylase. Mol Cell 23: 377-387.

7. Bolte S Cordelières FP (2006) A guided tour into subcellular colocalization analysis in light microscopy. J Microsc 224: 213-32.

8. Janke C, Bulinski JC (2011) Post-translational regulation of the microtubule cytoskeleton: mechanisms and functions. Nat Rev Mol Cell Biol 12: 773-786.

9. Cho HP, Liu Y, Gomez M, DunlapJ, Tyers M, et al. (2005) The dual-specificity phosphatase CDC14B bundles and stabilizes microtubules. Mol Cell Biol 25: $4541-4551$.

\section{Supporting Information}

Figure S1 Centaurin- $\alpha 2$ mRNA expression level in HeLa and H937 cell. Centaurin- $\alpha 2$ mRNA expression level in HeLa and H937 cell lines by real time RT-PCR normalized with the GAPDH transcript housekeeping. The level of GAPDH expression was comparable in all samples tested. Following the mean value of Centaurin- $\alpha 2$ expression level, calculated by GAPDH normalization and the estimation of $2^{-\Delta \mathrm{Ct}}$, is about 20 fold lower in HeLa than in H937 cell lines.

(TIF)

Figure s2 Centaurin- $\alpha_{2}$ colocalizes with MTs. Fluorograms showing the degree of colocalization between green (HA) and red $(\mathrm{MTs})$ signals in pCGN (pCGN) or pCGN-Centaurin- $\alpha_{2}$ (pCenta) expression.

(TIF)

Figure S3 Bait autoactivation assay. $\beta$-galactosidase assay of L40 yeast co-transformed with the bait construct pSTT91-centaurin- $\alpha_{2}$ and the pACT2 empty vector, and of the positive control L40 yeast transformed with the two known interacting proteins CoRest-Kia0601. The presence of blue colonies only in the control transformed L40-yeast indicates that the bait pSTT91centaurin- $\alpha_{2}$ does not auto-activate the $\beta$-galactosidase gene. (TIF)

\section{Acknowledgments}

The authors thank Dr. Milena Crippa, Dr. Sara Rigoletto for their support to yeast two hybrids experiments, Dr. Andrea Canuti for his support to biochemical analysis and Dr. Manuela Martinoli for her technical contribution to the transfected cells preparation.

\section{Author Contributions}

Conceived and designed the experiments: DC PR GC AA EB. Performed the experiments: PZ DC MS MV VP. Analyzed the data: PR GC DC EB MS PZ. Contributed reagents/materials/analysis tools: PR GC EB AA. Wrote the paper: PR GC DG MS PZ. Bioinformatic analysis: MV. Provided the bait cDNA library: EB. Yeast two hybrids assays: MS. Microtules, tubulin assays: DC. CEANTA2 overexpression/silencing evaluation on mitotic spindle formation: PZ. Centaurin alpha 2 purification: VP.

10. Geyp M, Ireland CM, Pittman SM (1996) Increased Tubulin acetylation accompanies reversion to stable ploidy in vincristine-resistant CCRF-CEM cells. Cancer Genet Cytogenet 87: 116-122.

11. Krauss SW, Spence JR, Bahmanyar S, Barth AI, Go MM, et al. (2008) Downregulation of protein $4.1 \mathrm{R}$, a mature centriole protein, disrupts centrosomes, alters cell cycle progression, and perturbs mitotic spindles and anaphase. Mol Cell Biol 7: 2283-94.

12. Sullivan KF, Cleveland DW (1986) Identification of conserved isotype-defining variable region sequences for four vertebrate B-Tubulin polypeptide classes. Proc Natl Acad Sci U S A 83: 4327-4331.

13. Nogales E, Wolf SG, Downing KH (1998) Structure of the alpha B-Tubulin dimer by electron crystallography. Nature 391:199-203.

14. Wade RH (2009) On and around microtubules: an overview. Mol Biotechnol 43: 177-191.

15. Stelzl U, Worm U, Lalowski M, Haenig C, Brembeck FH, et al. (2005) A human protein-protein interaction network: a resource for annotating the proteome. Cell 122: 957-968.

16. Stricker R, Chow KM, Walther D, Hanck T, Hersh LB, et al. (2006) Interaction of the brain-specific protein p42IP4/centaurin-alphal with the peptidase nardilysin is regulated by the cognate ligands of p42IP4, PtdIns $(3,4,5)$ P3 and Ins $(1,3,4,5) \mathrm{P} 4$, with stereospecificity. J Neurochem 98: 343-354.

17. Haase A, Nordmann C, Sedehizade F, Borrmann C, Reiser G (2008) RanBPM, a novel interaction partner of the brain-specific protein $\mathrm{p} 42$ (IP4)/centaurin alpha-1. J Neurochem 105: 2237-2248.

18. Venkateswarlu K, Brandom KG, Lawrence JL (2004) Centaurin-alphal is an in vivo phosphatidylinositol 3,4,5-trisphosphate-dependent GTPase-activating 
protein for ARF6 that is involved in actin cytoskeleton organization. J Biol Chem 279: 6205-6208.

19. Sedehizade F, Hanck T, Stricker R, Horstmayer A, Bernstein HG, et al, (2002) Cellular expression and subcellular localization of the human $\operatorname{Ins}(1,3,4,5) \mathrm{P}(4)$ binding protein, p42(IP4), in human brain and in neuronal cells. Brain Res Mol Brain Res 99: 1-11.

20. Chang JS, Kim SK, Kwon TK, Bae SS, Min DS, et al. (2005) Pleckstrin homology domains of phospholipase $\mathrm{C} \gamma 1$ directly interact with BTubulin for activation of phospholipase $\mathrm{C} \gamma 1$ and reciprocal modulation of $B$ Tubulin function in microtubule assembly J Biol Chem 280: 6897-6905.

21. Lemmon MA, Ferguson KM, Schlessinger J (1996) PH domains: diverse sequences with a common fold recruit signaling molecules to the cell surface. Cell 85: 621-624.

22. Hubbert C, Guardiola A, Shao R, Kawaquchi Y, Ito A, et al. (2002) HDAC6 is a microtubule-associated deacetylase. Nature 417: 455-458.

23. Reed NA, Cai D, Blasius TL, Jih GT, Meyhofer E, et al. (2006) Microtubule acetylation promotes kinesin-1 binding and transport. Curr. Biol 16: 2166-2172.

24. Venkateswarlu K, Hanada T, Chishti AH (2005) Centaurin-alphal interacts directly with kinesin motor protein KIF13B. J Cell Sci 118: 2471-2484.

25. Palazzo A, Ackerman B, Gundersen GG (2003) Cell biology: Tubulin acetylation and cell motility. Nature 421: 230.

26. Eot-Houllier G, Venoux M, Vidal-Eychenié S, Hoang MT, Giorgi D, et al. (2010) Plk1 regulates both ASAP localization and its role in spindle pole integrity. J Biol Chem 285: 29556-29568.

27. Lee J, Jeong Y, Jeong S, Rhee K (2010) Centrobin/NIP2 Is a Microtubule Stabilizer Whose Activity Is Enhanced by PLK1 Phosphorylation during Mitosis. J Biol Chem 285: 24576-25485

28. Nakamura M, Masuda H, Horii J, Kuma K, Yokoyama N, et al. (1998) When overexpressed, a novel centrosomal protein, RanBPM, causes ectopic microtubule nucleation similar to gamma-Tubulin. J Cell Biol 143: 1041-1052.

29. Brinkley BR (2001) Managing the centrosome numbers game: from chaos to stability in cancer cell division. Trends Cell Biol 11: 18-21.

30. Hinchcliffe EH, Sluder G (2001) Centrosome duplication: three kinases come up a winner! Curr Biol 11: R698-701.
31. Fukasawa K (2007) Oncogenes and tumour suppressors take on centrosomes. Nature Reviews Cancer 7: 911-924

32. Smith E, Héarat N, Veseky C, Rooseboom I, Larch C, et al. (2011) Differential control of Eg5-dpendent centrosomes separation by Plk1 and Cdk1. The EMBO J 30: 2233-2245.

33. Lingle WL, Lutz WH, Ingle JN, Maihle NJ, Salisbury JL (1998) Centrosome hypertrophy in human breast tumors: implications for genomic stability and cell polarity. Proc Natl Acad Sci U S A 95: 2950-2955.

34. Ghadimi BM, Sackett DL, Difilippantonio MJ, Schröck E, Neumann T, et al. (2000) Centrosome amplification and instability occurs exclusively in aneuploid, but not in diploid colorectal cancer cell lines, and correlates with numerical chromosomal aberrations. Genes Chromosomes Cancer 27: 183-190.

35. Bartelt-Kirbach B, Wuepping M, Dodrimont-Lattke M, Kaufmann D (2009) Expression analysis of genes lying in the NF1 microdeletion interval points to four candidate modifiers for neurofibroma formation. Neurogenetics 10: 79-85.

36. Denti S, Sirri A, Cheli A, Rogge L, Innamorati G, et al. (2004) RanBPM is a phosphoprotein that associates with the plasma membrane and interacts with the integrin LFA-1. J Biol Chem 279: 13027-13034.

37. Basu R Chang F (2007) Shaping the actin cytoskeleton using microtubule tips. Curr Opin Cell Biol 19: 88-94.

38. Al-Bassam J, Ozer RS, Safer D, Halpain S, Milligan RA (2002) MAP2 and tau bind longitudinally along the outer ridges of microtubule protofilaments. J Cell Biol 157: 1187-1196.

39. Cartelli D, Ronchi C, Maggioni MG, Rodighiero S, Giavini E, et al. (2010) Microtubule dysfunction precedes transport impairment and mitochondria damage in MPP+-induced neurodegeneration. J Neurochem 115: 247-58.

40. Castoldi M, Popov AV (2003) Purification of brain tubulin through two cycles of polymerization-depolymerization in a high-molarity buffer. Proteins Exp purify 32: 83-88.

41. Moncini S, Salvi A, Zuccotti P, Viero G, Quattrone A, et al. (2010) The Role of miR-103 and miR-107 in Regulation of CDK5R1 Expression and in Cellular Migration. PLoS ONE 6(5): e20038. doi:10.1371/journal.pone.0020038. 